\title{
Ueber den Werth des künstlichen Abortus.
}

\author{
Von \\ Dr. Cohnstein, \\ Docent an der Universität Berlin.
}

Den Werth der geburtshilflichen Operationen auf das richtige Maass zurickzufiuhren, ist die Aufgabe einer unbefangenen Kritik. Während die kiinstliche Frühgeburt in ihrer Berechtigung beim engen Becken durch Spiegelberg s statistische Arbeit eine gewisse Beschränkung erfahren hat, und in ihrer Empfehlung bei Krankheiten der Mutter immer mehr an Anhang einbüssen muss, sofern man genall die Resultate für Mutter und Kind bei dieser Operation mit anderweitigen Behandlungsmethoden - chirurgischen Operationen - vergleicht, schleppen sich beim kiinstlichen Aborte die Indicationen traditionell von Geschlecht zu Geschlecht fort. In Deutschland stehen sie allerdings nur auf dem Papier, wir finden sie in den Lehrbiichern wohl besprochen und präcisirt, während die Praxis sich meist abweisend ihnen gegenüber verhält. Anders ist es in Frankreich, wo vorwiegend das unstillbare Erbrechen als inäufigste Veranlassung für den künstlichen Abort acceptirt.ist. Summiren wir die von Guéniot, Dubois, Cazeaux, Aubenas, Herrgott, Caradec, Bourdon, Chomel, Griolet, Raimbert, Pigeolet, Pétrequ'in, Carter, Fevron, Verdalle, Notta publicirten Beobachtungen, die sich nahezu auf zweihundert belaufen, so steht uns zur Eruirung speciell dieser Indication ein ausreichendes Material zu Gebote.

Bricht die Schwangere zu jeder Zeit Alles, was sie geniesst, aus, leidet das Allgemeinbefinden und insbesondere der Kräftezustand, sind die allgemeinen diätetischen Vorschriften ebenso erfolglos, wie die Medication, welche bekanntlich durch Herabsetzung der Erregbarkeit des Magens oder durch Abstumpfung des gesammten sensiblen Nervensystems einzuwirken versucht, so wird das Erbrechen als unstillbares bezeichnet.

Den Beweis, dass der Abort Hilfe gegen das unstillbare Erbrechen gewährt, lieferten die Beobachtungen, in welchen nach Ausstossung der Frucht das Erbrechen aufhörte. Sie animirten auch zum künstlichen Eingriff, sofern die Expulsion nicht spontan eintrat.

Fragen wir zuerst, welchen Ausgang das in Rede stehende Leiden ninme, wenn die Operation unterbleibt, so haben wir, der Hänfigkeit nach, eine spontane Besserung, den spontanen Abort, in dritter Reihe erst den Tod durch Marasmus, intermittirendes Fieber, Peritonitis zu vermerken. Dieser Punkt verdient unsere Beachtung, 
insofern wir erfahren, dass die Prognose quoad vitam bei einer exspectativen Therapie keine ganz nngünstige ist.

Spontane Besserung erfolgt gewöhnlich dann, wenn das Erbrechen in den späteren Monaten, so im 4. und 5. zuerst aufgetreten ist. Je mehr wir uns der Schwangerschaftsmitte nähern, um so häufiger können wir günstige Resultate erwarten. Zeigt sich das Erbrechen schon in den ersten 3 Monaten, so ist der Verlauf bei Erst- und Mehrgebärenden nicht gleich. Während das Erbrechen bei den letzteren relativ nicht häufig einen bedenklichen Grad erreicht, sind die Chancen bei Erstgebärenden weit schlechter.

Was nun den Abort im Allgemeinen betrifft, so hört in ungefähr 40\% der Fälle, nach Ausschliessung der Frucht,' das Erbrechen sofort und vollständig auf. Hierbei müssen wir aber den künstlichen und den spontanen Abort auseinanderhalten, weil an den $40 \%$ dieser mit 25 , jener mit nur $15 \%$ participirt. Die restirenden $60 \%$ umfassen die Fälle, in welchen das Erbrechen nur unvollkommen nachgelassen $(26 \%)$, in welchen es überhaupt nicht aufgehört $(18 \%)$, in welchen es stärker aufgetreten $(4 \%)$ und in denen gleichzeitig oder kurze Zeit nach dem Aborte der Tod (12\%) erfolgt ist. Auch hier deuten die Zahlenverhältnisse darauf hin, dass die Prognose günstiger, wenn die Geburt spontan eintritt, als wenn sie künstlich hervorgerufen wird. Im letzteren Falle nämlich verläuft die Geburt weit langsamer, es kommt häufiger zur Retention von Eihautresten und Placentarstücken, starken Blutungen, Tetanus, Para- und Perimetritis. Auch bei der Frühgeburt ist die Prognose bekanntlich günstiger, wenn sie spontan eintritt, als wenn sie künstlich hervorgerufen wird.

Der Vorwurf der Kritik, man könne aus derartigen Beobachtungen keine massgebenden Schlüsse ziehen, weil die günstig verlaufenen Fälle veröffentlicht, während die ungünstigen verschwiegen werden, trifft hier am allerwenigsten zn, wo die ungünstigen Resultate die günstigen so bedeutend überragen.

Abstrahiren wir von anderen Schlussfolgerungen, so verdient doch das Ergebniss unsere volle Beachtung, dass der künstliche Abort gegen das unstillbare Erbrechen nur in einer relativ kleinen Anzahl von Fällen sichere Hülfe gewährt.

Behaupten die Anhänger des künstlichen Aborts, dass in den ungünstig verlaufenen Fällen zu spät operirt worden sei, dass man nicht abwarten dürfe, bis Fieber eingetreten und die Entkräftung einen bedenklichen Grad angenommen hat, so ist dieser Einwand insofern gewiss gerechtfertigt, als nach Analogie aller anderen Operationen auch beim künstlichen Abort nur dann günstige Resultate erwartet werden können, wenn rechtzeitig operirt wird. Der Rückschluss ist aber nicht stichhaltig, dass, weil dem Abort der 'Tod gefolgt ist, die Operation zu spät ausgeführt worden ist. Wohl könnte ich die hierher gehörigen Fälle von Dubois, Cazeaux, Churehill, welche lethal verlaufen sind, als Gegenbeweis vorbringen, wenn ich nicht dem Vorwurf zu begegnen fürchten müsste, dass auch von diesen anerkannt bewährten Geburtshelfern der Abort zu spät provocirt worden sei. 
Nehmen wir aber die Beobachtungen, in welchen das Erbrechen nach Ausstossung der Frucht nicht nachgelassen, in welchen es stärker aufgetreten ist, in denen der tödtliche Ausgang sogar beschleunigt worden, so muss es einleuchten, dass die günstige Meinung vom künstlichen Abort beim unstillbaren Erbrechen ïbertrieben worden ist.

Die Aetiologie des Leidens ist noch wenig erforscht. Bisweilen trifft man Fraten, die schon vor der Schwängerung häufig an Erbrechen gelitten haben; in anderen Fällen begegnet man Individuen, die bis zum Eintritte der Gravidität vollkommen gesund waren und nun unter dem Einflusse der veränderten Blutmischung laboriren. Die Sectionen ergaben hin und wieder wohl gröbere pathologischanatomische Veränderungen der Magenwände, häufig aber auch nichts weiter, als das Bild einer verbreiteten Anämie.

Was die Ursachen des spontanen Aborts betriff, so wollen wir nicht bestreiten, dass die durch das häufige Erbrechen reducirte Ernährung der Frucht ins Gewicht fallen kann. Wesentlicher sind die Brechbewegungen, bei welchen es durch Abflachung des Zwerchfelles und Spannung der Bauchmuskeln zu einer bedeutenden Verengung des Bauchraums und Compression des Uterus kommt. Es tritt hinzu die am Ende der Brechanfälle erfolgende Drucksteigerung im Aortensysteme (Traube), welche die Zerreissung der Verbindungen des Eies begünstigt.

Treten bei den schon heruntergekommenen Schwangeren noch profuse Blutungen in partu auf, so kann der tödtliche Ausgang' beschleunigt werden. Ist die Metrorrhagie bei der Geburt aber nur mässig, die Kranke noch halbwegs bei Kräften, so kann der Abort Besserung oder vollständigen Nachlass des Erbrechens erwirken. In der Casuistik findet sich eine nicht geringe Anzahl von Fällen, in welchen dieser günstige Ausgang anf die raschen und bedeutenden Veränderungen zurïekzuführen ist, welche der Gesammtorganismus im Wochenbett überhaupt erfährt.

Während sich therapeutisch der künstliche Abort also nur ausnahmsweise verwerthen lassen wird, fehlen uns zur spontanen Ausstossung der Frucht alle Mittel und Wege. Es fragt sich nun, worin die Behandlung beim unstillbaren Erbrechen bestehen soll? Berücksichtigen wir, dass Brechbewegungen zumeist dann eintreten, wenn durch Zufuhr von Nahrung per os die Vagusendigungen in der Magenschleimhaut erregt werden, dass die Prognose keine ganz unguinstige ist, wenn das Leiden sich selbst überlassen bleibt, und sich relativ bessert, sobald die Schwanger'schaft weiter vorgeschritten ist, dass der Tod am häufigsten unter dem Bilde der Inanition eintritt, so werden wir die Schwangerschaft unberührt lassen und nur dafür Sorge tragen, dass vom Körper genügende Nahrungsmengen unter Ausschluss des Magens verdaut werden. Ermöglichen wir die Ernährung der Kranken, bis die Schwangerschaft die ersten Monate überschritten hat, so haben wir Aussicht, die Patientin zu erhalten; tritt:während dieser diätetischen Behandlung Ábort ein, so trifft er eine durchaus 
nicht entkräftete Patientin, welche die Blutungen bei der Geburt auch überstehen wird.

Es ist constatirt, dass der Dickdarm Nahrungsklystiere zu resorbiren im Stande ist und dass durch diese künstliche Ernährung Kranke wochenlang erbalten werden können. Die Möglichkeit der Ernährung per anum ist bei Schwangeren nicht schwieriger, als bei Nichtschwangeren.

Im vorigen Jahre lernte ich in Blankenberghe eine junge Erstgebärende im 3. Honat ihrer Gravidität kennen, die zur Beseitigung eines hartnäckigen, seit Wochen andauernden Erbrechens an die See geschickt war. Die Veränderung des Klimas und die Seeluft schienen in den ersten Tagen das Uebel abschwächen zu wollen. Bald trat das Erbrechen von Neuem auf, wurde constant und hartnäckiger als friiher, so dass der Magen nichts behielt. Die von Hause aus mitgenommenen Medicamente leisteten gar nichts, auch die von Dr. van Mullem gereichten Narcotica in kleiner und grosser Dosis blieben ganz erfolglos. Die Rückreise konnte wegen der schnell überhand genommenen Entkräftung nicht ausgeführt werden. Zur Beseitigung der Obstipation von mir verordnete grosse Klysmata von Seewasser hatten voriibergehenden Erfolg. Die Patientin ist anämisch, schlecht ernährt, psychisch sehr erregt, bricht auf jede Nahrungszufuhr per os, wird in den Pausen von Sodbrennen, Aufstossen gequält, klagt über Kopfschmerzen, Uebelkeit, Druck und Schmerzgefühl in der ganzen oberen Bauchgegend; Schlaf relativ gut, nur durch den Durst unterbrochen. Der Puls ist klein, mässig frequent, die Respiration normal, die Zunge rein, starker Foetor ex ore, der Uterus vergrössert und normal anteflectirt. Da es misslich.ist, den künstlichen Abort in einem Badeorte auszufuihren, in welchem man keine Befugniss zur Praxis hat, recurrirte ich auf dieses Rettungsmittel nicht weiter, sondern verordnete Klysmata nutrientia von reiner Milch, von Bouillon mit Eigelb, welche mittels eines Eguisier'schen Irrigateurs dreimal täglich lauwarm eingebracht wurden. Die ersten beiden Nahrungsklystiere gingen nach kurzer Zeit wieder ab, das dritte und die folgenden blieben; intercurrent zeigte sich wohl Diarrhöe, die von selbst nachliess. Reinigungsclysmata waren in der ersten Zeit nicht nöthig. Das Erbrechen hielt in der ganzen ersten Woche dieser Behandlung noch an, kehrte von da ab in grösseren Pausen wieder, als jede $\mathrm{Zu}^{-}$ fuhr von Nahrung per os, jeder Schluck Wasser, selbst das Mundausspülen untersagt war. Die Mundhöhle wurde einigemal des Tages mit einem in kaltes Wasser getanchten Läppchen gereinigt. Unter dieser Behandlung besserte sich der Kräftezustand, der Puls wurde voller. Wie ich später hörte, war vier Wochen nach Beginn dieser diätetischen Kur die Patientin im Stande, die Rïckreise anzutreten. Am normalen Schwangerschaftsende erfolgte die Geburt eines lebenden Kindes.

Ein zweiter Fall von unstillbarem Erbrechen ist mir seit der Zeit nicht vorgekommen. Bei hartuäckigem Erbrechen Schwangerer abstrahire ich aber von jeder Behandlung und verordne mit Vorliebe 
die von Leube (Deutsches Archiv f. klinische Med. X. S. 1-54) empfohlene Injection eines aus fein zerhacktem Fleisch und fein zerhackter Pancreassubstanz bestehenden Gemisches in den durch Wasserclysmata vorher gereinigten Mastdarm. Ich kann versichern, dass sich unter dieser Behandlung das Allgemeinbefinden bessert und das Erbrechen nachlässt, sofern die Nahrungszufuhr per os ganz sistirt, der Magen fiir eine Zeit lang ganz ausser Thätigkeit gesetzt wird. -

Eine weitere Indication für den künstlichen Abort bildet das absolnt verengte Becken. Im Hinblick auf die unguinstigen Resultate beim Kaiserschnitt und der Perforation erachtete William Cooper den Abort für berechtigt. Sein Vorschlag fand in England, Frankreich, Deutschland Anklang. Nachdem sich aber in den letzten Jahren die Ueberzeugung Bahn gebrochen hat, dass die Prognose beim Kaiserschnitt und der Perforation sich wesentlich besser gestaltete, wenn zur rechten Zeit und unter günstigen äusseren Umständen operirt wird, ist der Abort für solche Fälle reservirt worden, in welchen die Schwangeren den Kaiserschnitt voranssichtlich nicht überstehen werden. Diese Indieation ist durchaus nicht erschöpft, so lange der Nachweis fehlt, wie sich das Sterblichkeitsverhältniss der Mütter beim künstlichen Abort, gegeniiber dem Kaiserschnitt, stellt; denn die Annahme, dass beim künstlichen Abort das Leben der Mutter nicht in Gefahr kommt, ist ganz und gar nicht richtig. Nach den vielen Debatten, die über diese Indication geführt worden sind, hätte man meinen müssen, dass ein reiches Material von Beobachtungen vorhanden ist. Dem ist nicht so; in der Literatur finden sich nur sehr vereinzelte Fälle. War der Ausgang für die Mütter in den uns bekannten 10 Fällen von künstlichem Abort bei Lenoir (Bullet. de l'Acad. XVII. 9-13. 1852), Laborie ('Union méd. 1848, S. 94), Tissier (l'Jnion méd. 1868, S. 88), Kuh n (Wien. med. Wochensch. 1863, S. 150), Whitehead (Lond. med. Gaz. 1841. Septbr. S. 938 u. 971) günstig, so können wir aus naheliegenden Gründen die Indication deshalb noch nicht für begründet erachten.

Soweit ich mich aus der Literatur informiren konnte, entspricht der zur Erfüllung einer Indicatio vitalis bei Herz- und Lungenkrankheiten empfohlene Abort den gehegten Erwartungen nicht. 